\title{
The changing epidemiology of pneumococcal diseases: new challenges after widespread routine immunization
}

\author{
Peter Francis Raguindin ${ }^{1,2}$ (D)
}

Received: 18 January 2020 / Revised: 20 May 2020 / Accepted: 27 May 2020 / Published online: 12 June 2020

(C) Swiss School of Public Health (SSPH+) 2020

Though the use of the pneumococcal conjugate vaccines (PCVs) has reduced the incidence of pneumonia and invasive diseases around the world, widespread vaccine use has resulted in serotype replacement (Balsells et al. 2017). Serotype replacement describes the shifting burden of invasive pneumococcal disease, from vaccine serotypes to other serotypes not covered by the vaccine, which may, paradoxically, increase disease burden (Weinberger et al. 2011). This has created new challenges like detecting nonvaccine pneumococcus strains, identifying changes in disease transmission patterns, and raised questions about adapting immunization strategies.

Pneumococcal diseases are most commonly caused by 10 to 13 bacterial serotypes, and current vaccines are designed to work against these. The remaining 80 serotypes are usually considered rare, but after widespread vaccine use, they are seen more often. The best serotype test is time-consuming and expensive, requires highly trained technical stave, and is difficult to scale up to process large numbers of samples (Geno et al. 2015). This results in less testing, so the true burden of non-vaccine serotypes is unknown. Recently, the field of pneumococcal diagnostics has shifted towards molecular-based techniques utilizing the voluminous data from the Global Pneumococcus Sequencing Project (https://www.pneumogen.net/gps/). Multiplex-PCR tests are especially promising because they

This Editorial is part of the series "Young Researcher Editorial", a training project of the Swiss School of Public Health.

Peter Francis Raguindin

pnraguindin@up.edu.ph

1 Institute of Social and Preventive Medicine, University of Bern, Mittlestrasse 43, 3027 Bern, Switzerland

2 Institute of Child Health and Human Development, National Institutes of Health, University of the Philippines Manila, Rm. 112 G/F National Institutes of Health Bldg., UP Manila, 623 Pedro Gil St. Ermita, 1000 Manila, Philippines can identify non-vaccine serotypes, are easy to use, and samples can be processed in huge batches (Jauneikaite et al. 2015).

The transmission pattern of pneumonia is also changing. Pneumococcus colonizes the nasopharynx, while 30-80\% of healthy children carry the bacteria, (Usuf et al. 2014) and colonization rates are highest in developing countries (Usuf et al. 2014). Since children are known reservoirs for disease-causing serotypes, controlling infection in this age group is crucial to public health. In countries with mature immunization programs, the vaccine serotypes are rarely seen in infants and younger children and pneumonia incidence has declined $22 \%$ across the population (McAllister et al. 2019). Nonetheless, in some countries, such as the UK, a recent epidemiologic assessment found increased incidence of invasive diseases not covered by the vaccine (Ladhani et al. 2018). The increase in disease burden among the elderly was $4 \%$ after 10 years of sustained high childhood immunization coverage (Ladhani et al. 2018). This is a group not targeted by routine PCV immunization, but was expected to benefit from high immunization coverage in children through herd immunity.

Our understanding that pneumococcal disease is controlled through the herd effect has been thrown into question. In most high-income countries, the herd effect becomes almost immediately apparent after the vaccine is introduced, but in developing countries the effect is more gradual (International Vaccine Access Center 2017). In high-income countries, serotype replacement seems to erode the effectiveness of a vaccine after long-term use, illustrated by the growing number of cases of invasive disease in adults. It is too early to tell if this is the case in developing countries since the vaccine was newly introduced and the program has not matured (International Vaccine Access Center 2017). We also do not know if transmission patterns will change since, as yet, there is little adult nasopharyngeal data from developing countries. We can expect more data in the future because many 
countries have set up nationwide surveillance monitoring of the post-routine immunization period.

We need more studies to determine the optimal vaccine schedule for pneumococcal vaccines, as the Strategic Advisory Group of Experts for Immunization pointed out in their latest meeting (October 2017) (World Health Organization 2017). All countries have chosen different vaccine products, targeted different age groups, and has established campaigns or phased-introduction and/or varying schedules. Given the wide variation in routine immunization programs in different countries, it is difficult to evaluate specific outcomes of interest like nasopharyngeal carriage, herd immunity, duration of protection, and transmission dynamics (International Vaccine Access Center 2017; Shiri et al. 2017). However, policymakers need to know the optimal dosing schedule so they can set the number of doses and shot schedules, as these factors guide implementation and determine costs for immunization programs.

The epidemiology of pneumococcal diseases will change as the pathogen evolves in response to vaccineinduced population immunity. Non-vaccine serotypes will likely dominate transmission cycles among carriers. When non-vaccine pneumococci become more invasive, we will see a higher proportion of them in those who present with the disease. Newer serotypes will also evolve in response to vaccine-induced herd immunity, raising new challenges for serotyping testing, for predicting disease transmission patterns and optimizing immunization schedules. All these challenges must be met if we want vaccination programs to provide continued benefits to the population.

Funding None.

\section{Compliance with ethical standards}

Conflict of interests The author declares that they have no conflict of interests.

\section{References}

Balsells E, Guillot L, Nair H, Kyaw MH (2017) Serotype distribution of Streptococcus pneumoniae causing invasive disease in children in the post-PCV era: a systematic review and metaanalysis. PLoS ONE 12(5):e0177113

Geno KA, Gilbert GL, Song JY, Skovsted IC, Klugman KP, Jones C et al (2015) Pneumococcal capsules and their types: past, present, and future. Clin Microbiol Rev 28(3):871-899

International Vaccine Access Center (2017) Pneumococcal conjugate vaccine review of impact evidence: summary of findings from systematic review. Johns Hopkins Bloomberg School of Public Health, Baltimore

Jauneikaite E, Tocheva AS, Jefferies JM, Gladstone RA, Faust SN, Christodoulides M et al (2015) Current methods for capsular typing of Streptococcus pneumoniae. J Microbiol Methods 113:41-49

Ladhani SN, Collins S, Djennad A, Sheppard CL, Borrow R, Fry NK et al (2018) Rapid increase in non-vaccine serotypes causing invasive pneumococcal disease in England and Wales, 2000-17: a prospective national observational cohort study. Lancet Infect Dis 18(4):441-451

McAllister DA, Liu L, Shi T, Chu Y, Reed C, Burrows J et al (2019) Global, regional, and national estimates of pneumonia morbidity and mortality in children younger than 5 years between 2000 and 2015: a systematic analysis. Lancet Glob Health 7(1):e47-e57

Shiri T, Datta S, Madan J, Tsertsvadze A, Royle P, Keeling MJ et al (2017) Indirect effects of childhood pneumococcal conjugate vaccination on invasive pneumococcal disease: a systematic review and meta-analysis. Lancet Glob Health 5(1):e51-e59

Usuf E, Bottomley C, Adegbola RA, Hall A (2014) Pneumococcal carriage in sub-Saharan Africa-a systematic review. PLoS ONE 9(1):e85001

Weinberger DM, Malley R, Lipsitch M (2011) Serotype replacement in disease after pneumococcal vaccination. Lancet 378(9807):1962-1973

World Health Organization (2017) Meeting of the strategic advisory group of experts on immunization, October 2017-conclusions and recommendations. Wkly Epidmiol Rec 92(48):729

Publisher's Note Springer Nature remains neutral with regard to jurisdictional claims in published maps and institutional affiliations. 